\title{
INDICE CLGP I.1.2.1
}

Prefazione $\quad$ V

Criteri editoriali $\quad$ VI

Revisori dei papiri $\quad$ IX

Siglorum et compendiorum explicatio $\quad X$

Conspectus librorum XII

ALCMAN 3

1a - P.Oxy. XXIV 2389. Kommentar zu Alkmans 1. Parthenion 11

1b - P.Oxy. XLV 3210. Kommentar zu Alkman? 47

2 - P.Oxy. XXIV 2390. Kommentar zu mindestens zwei

Liedern Alkmans und Liedern anderer Autoren (?) 54

3 - P.Oxy. XXIV 2391. Kommentar zu lyrischer Dichtung

in dorischem Dialekt, möglicherweise Alkman 86

4 - P.Oxy. XXIV 2392. Kommentar eines Dionysios zum 4.

Buch der Lieder des Alkman (eher ein Buchtitel als ein Sillybos) 102

5 - P.Louvre E 3320 (= P.Paris 71). Parthenion mit Randscholien (PMG 1) 106

6 - P.Oxy. XXIV 2387. Parthenion mit Randscholien (PMG 3) 128

7 - P.Congr.XV 1. Verse des Alkman mit Lesezeichen und einem Randscholion 136

8 - P.Oxy. XXIV 2393. Glossar, wahrscheinlich zum 2. Lied im 1. Buch der Lieder des Alkman 139

9 - P.Oxy. XXIX 2506, Frg. 1, 4, 5 und 17.

Kommentar zu lyrischen Dichtern, auch Alkman

10 - P.Oxy. XXIV 2394. Anmerkungen zu einem chorlyrischen Text im dorischen Dialekt (Alkman?)

Schede

$\begin{array}{ll}\text { (a) P.Oxy. XXIV } 2388 & 157\end{array}$

(b) P.Oxy. XXXVII $2802 \quad 157$

$\begin{array}{ll}\text { (c) P.Oxy. L } 3542 & 158\end{array}$

$\begin{array}{ll}\text { Index papyrorum } & 161\end{array}$

Index tabularum 162 

TAB. I

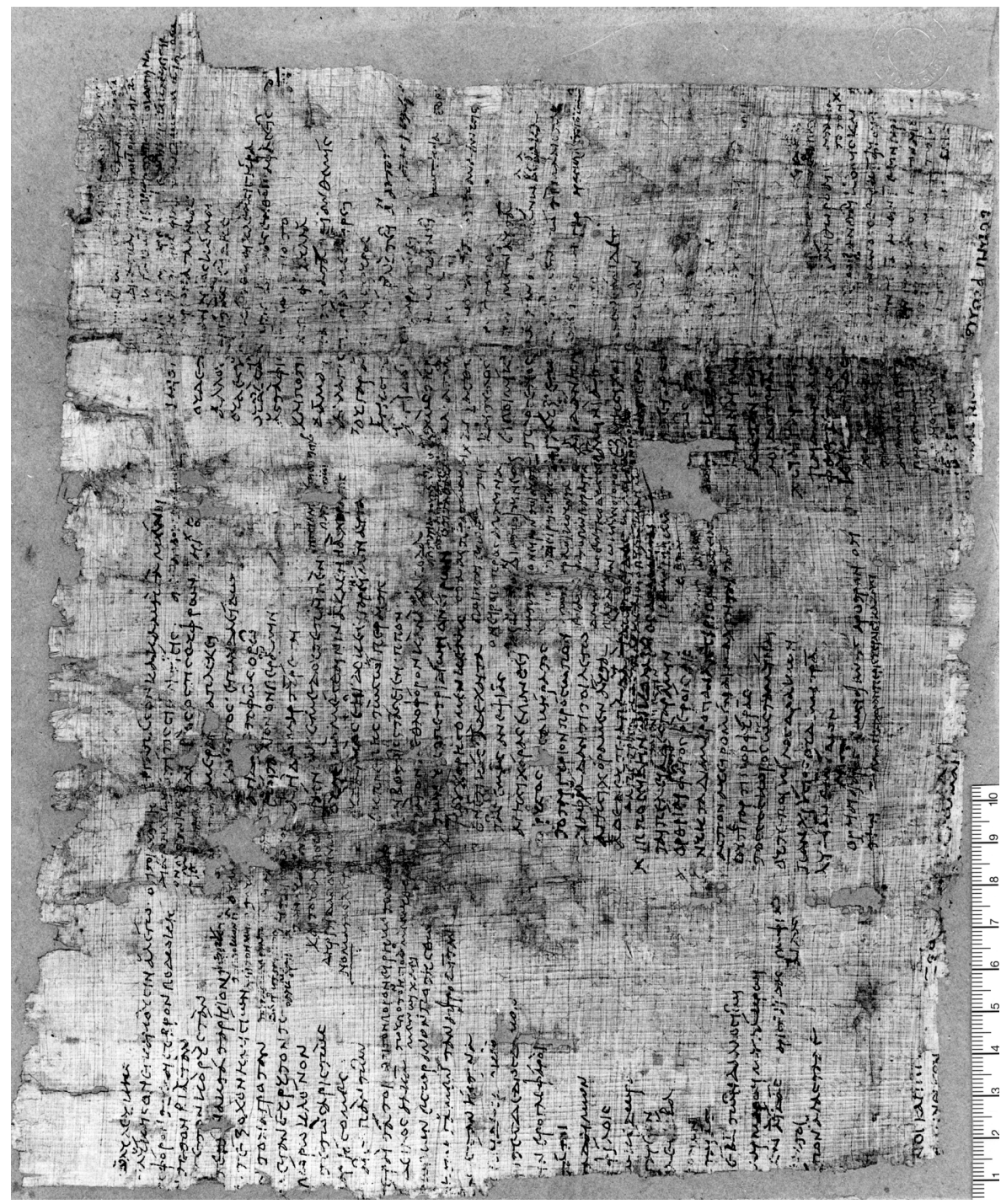

P.Louvre E $3320(=$ P.Paris 71) $\Rightarrow$ Alcman 5 


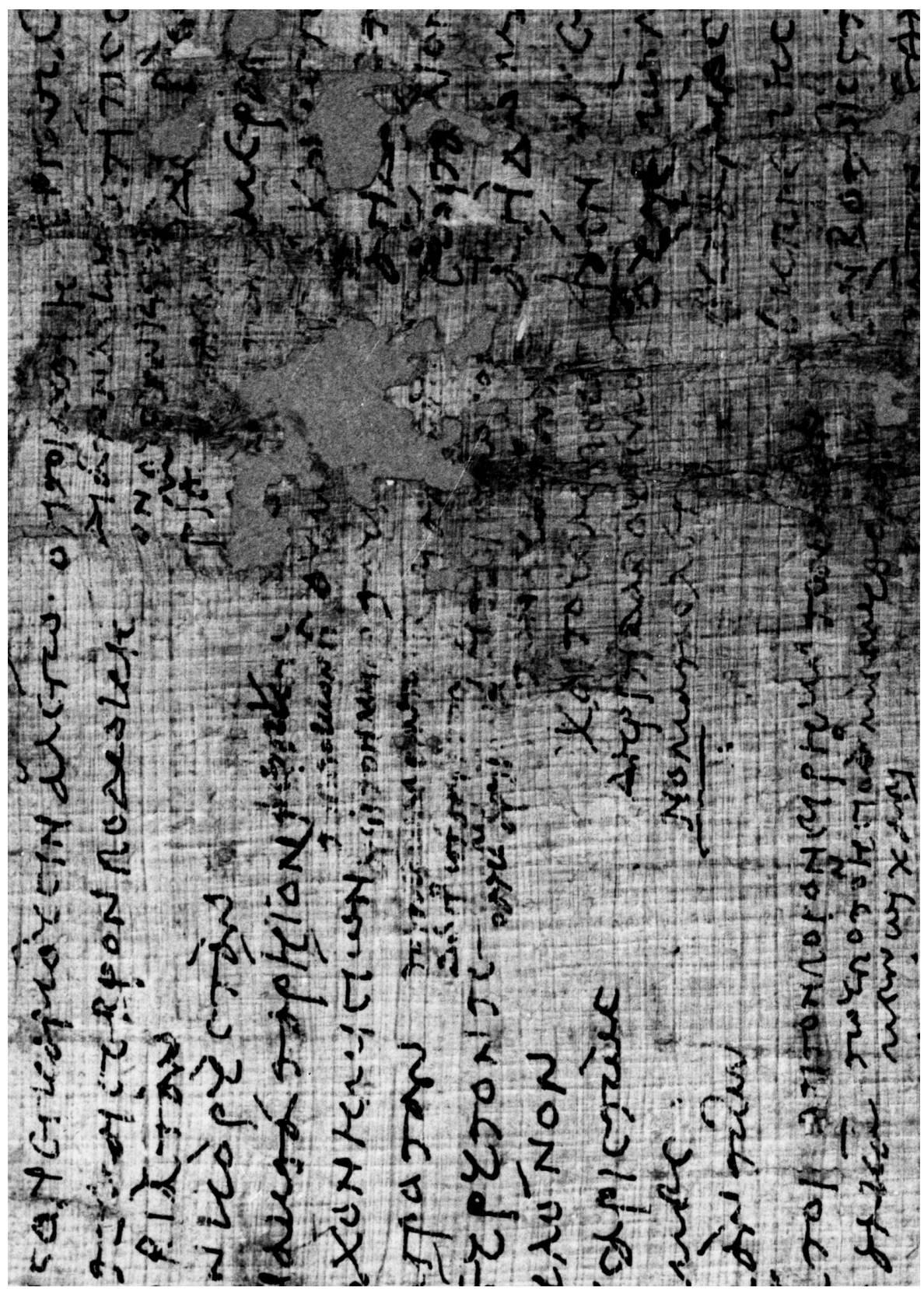

P.Louvre E 3320 (= P.Paris 71), ad VV. 2, 6, $14 \Rightarrow$ Alcman 5 
TAB. III

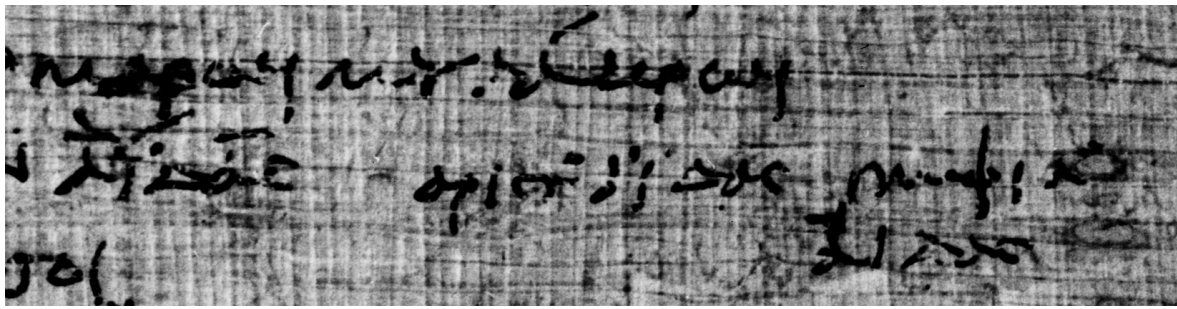

(a) P.Louvre E 3320 (= P.Paris 71), ad V. $32 \Rightarrow$ Alcman 5

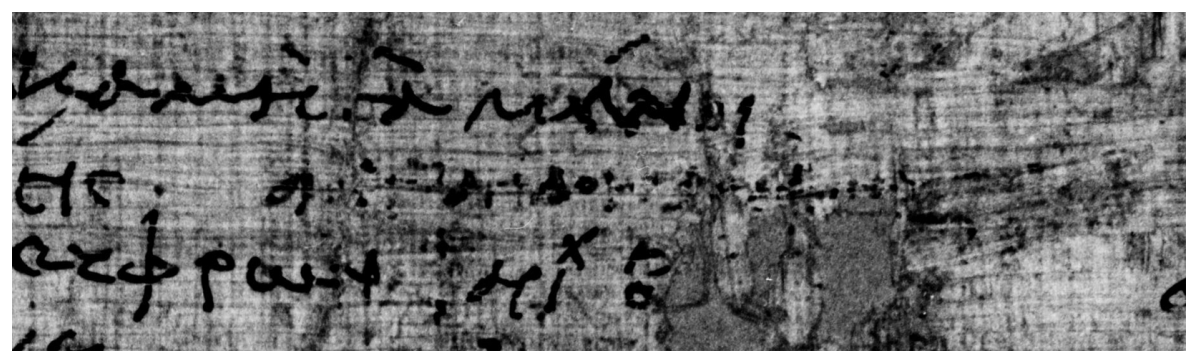

(b) P.Louvre E 3320 (= P.Paris 71), ad VV. 36, 37 A Alcman 5

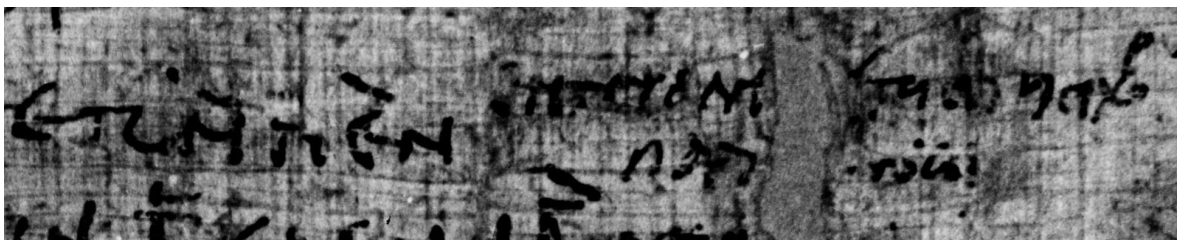

(c) P.Louvre E 3320 (= P.Paris 71), ad V. $43 \Rightarrow$ Alcman 5

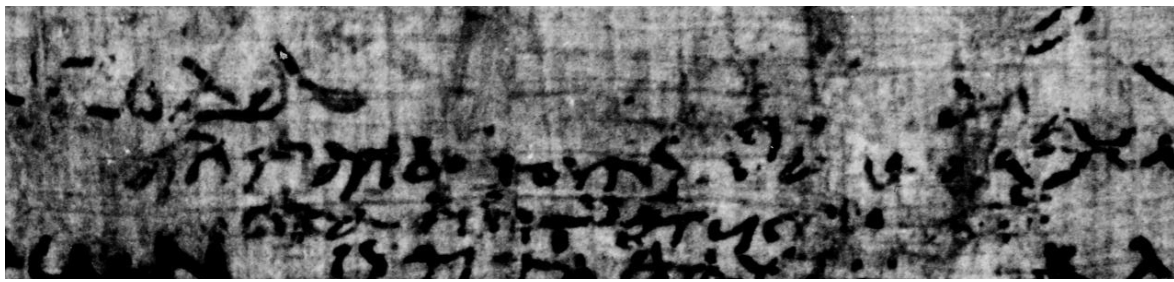

(d) P.Louvre E 3320 (= P.Paris 71), ad V. $48 \Rightarrow$ Alcman 5 


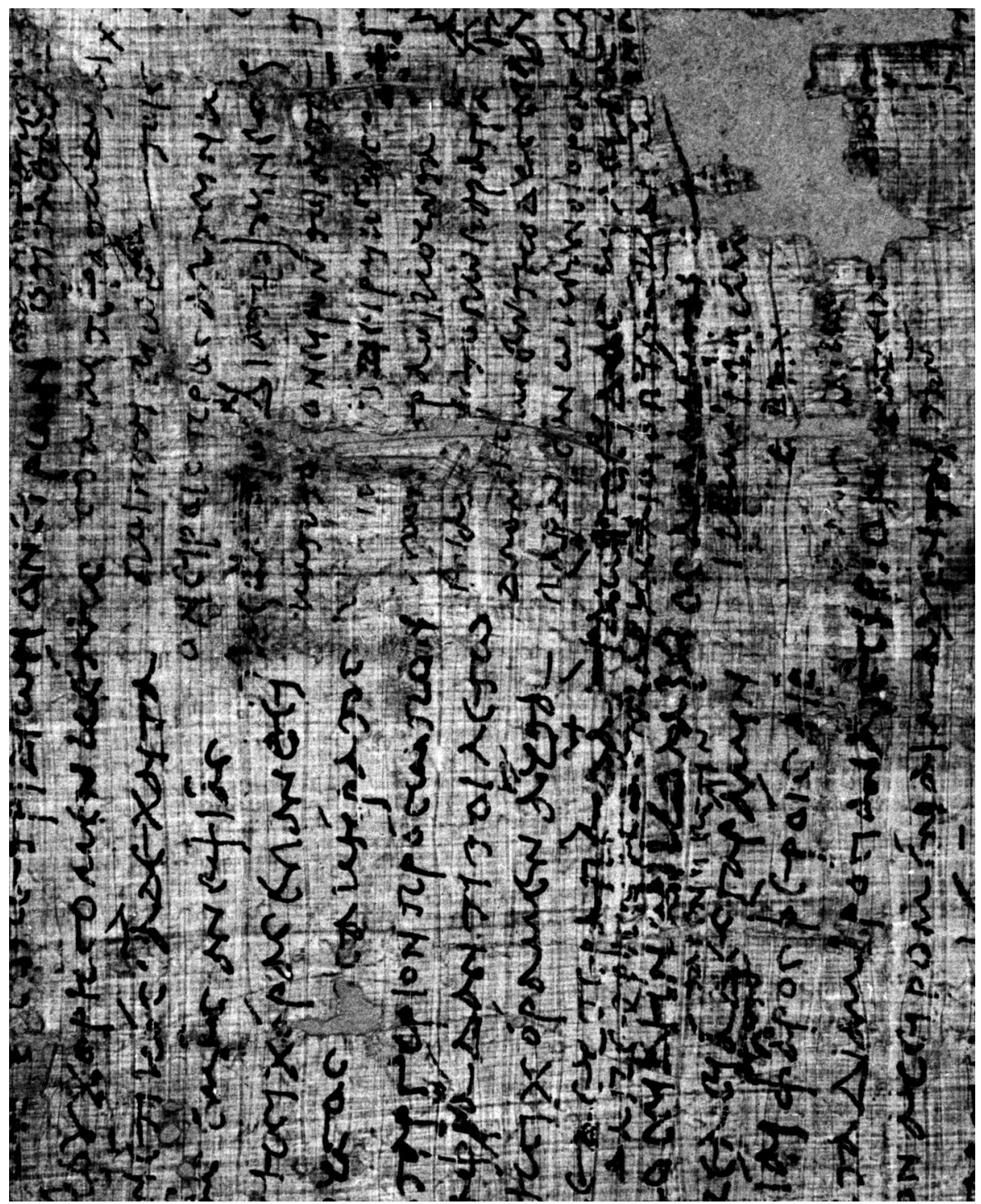

P.Louvre E 3320 (= P.Paris 71), ad VV. 49, 59, 61 (inter lineas), signum ad V. 87 scriptum $\Rightarrow$ Alcman 5 

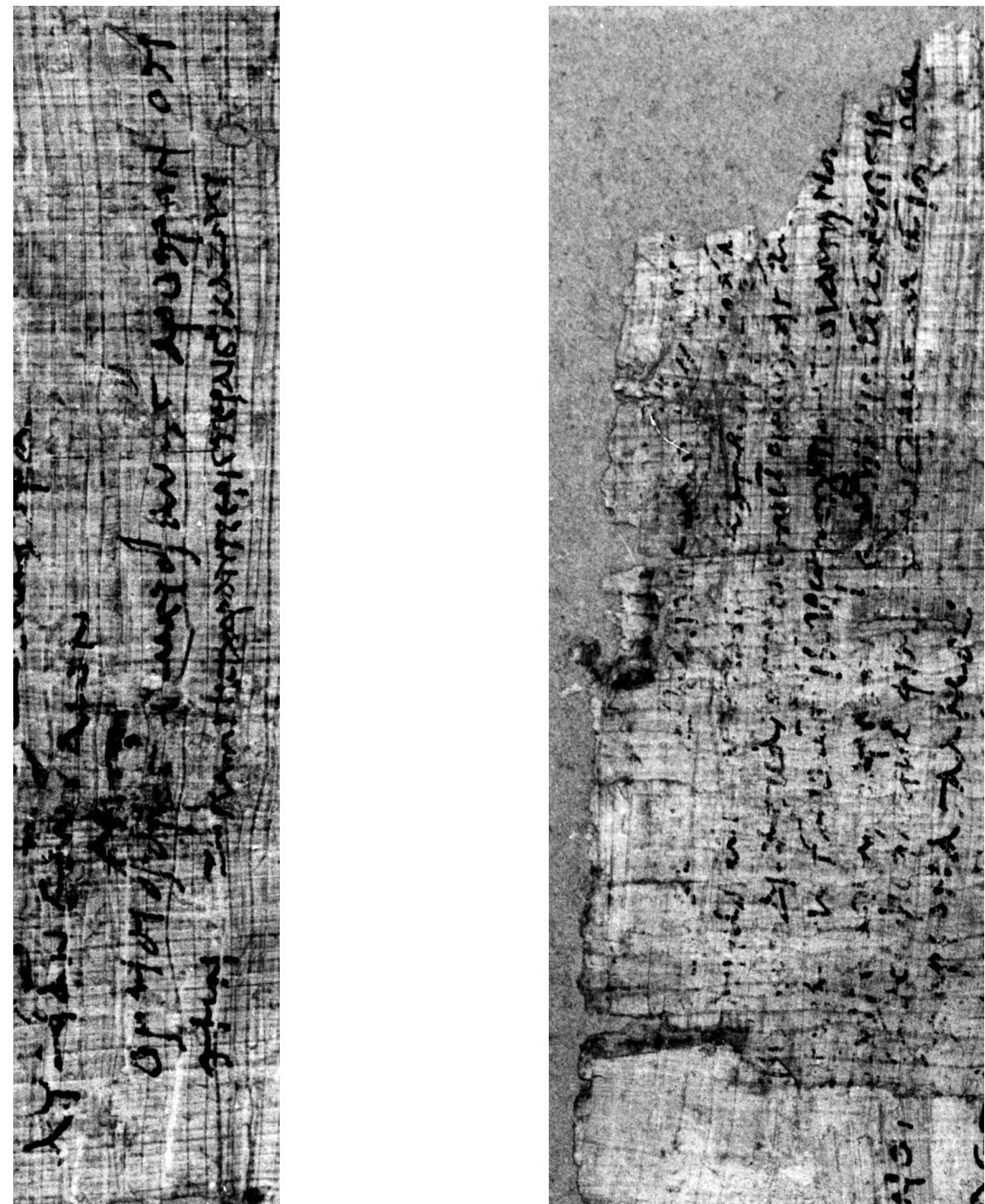

(a) P.Louvre E 3320

(b) P.Louvre E 3320

(= P.Paris 71),

$(=$ P.Paris 71)

ad V. $61 \Rightarrow$ Alcman 5

ad V. $70 \Rightarrow$ Alcman 5 
TAB. VI

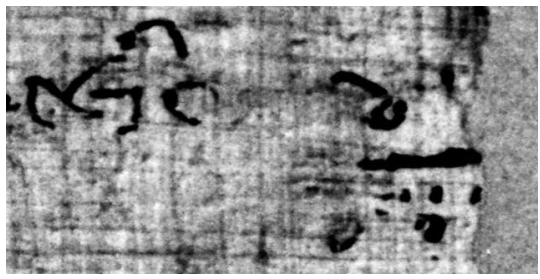

(a) P.Louvre E 3320 (= P.Paris 71), ad V. $105 \Rightarrow$ Alcman 5

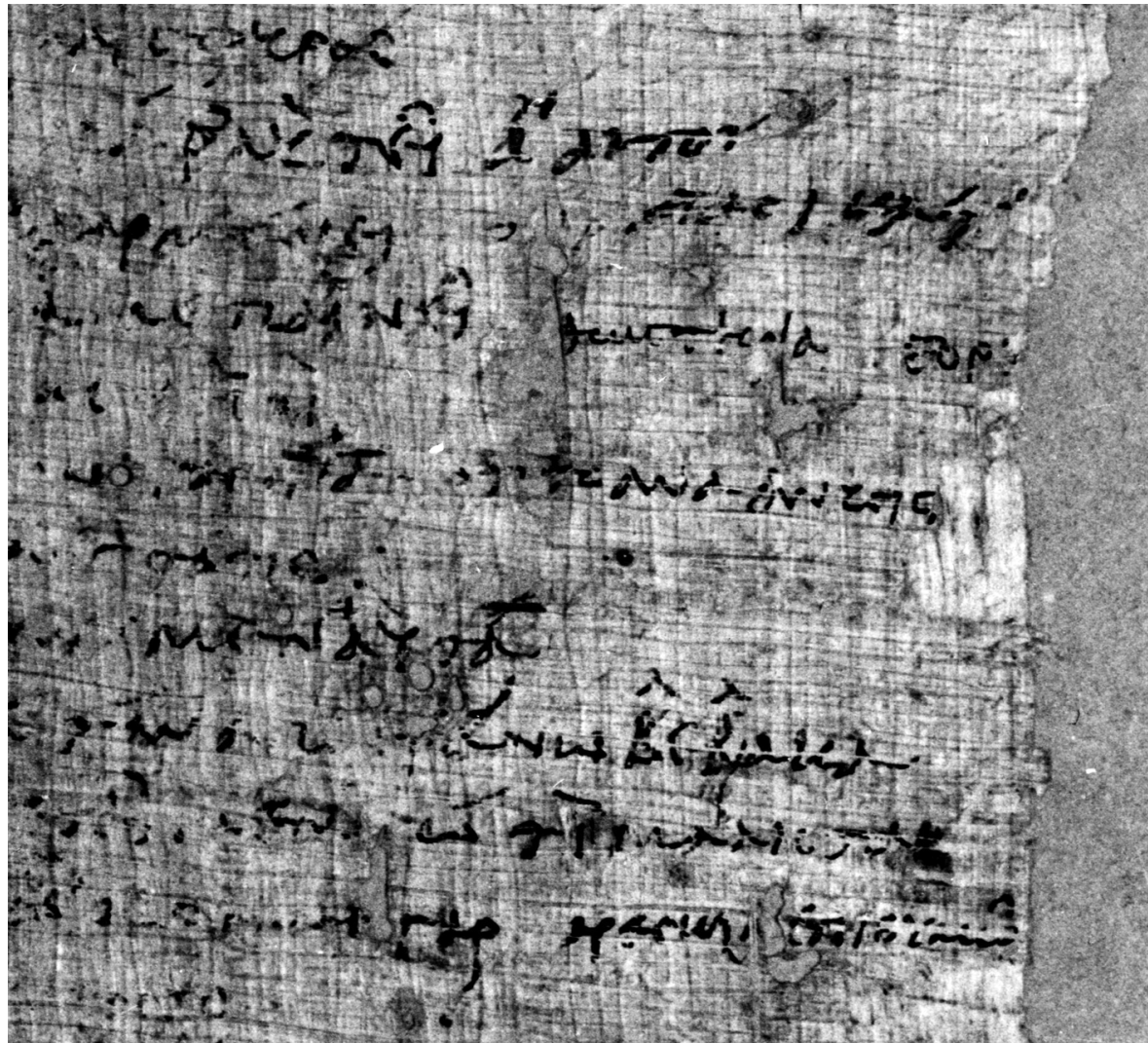

(b) P.Louvre E 3320 (= P.Paris 71), ad VV. 79, 81, 83, 88 $\Rightarrow$ Alcman 5 
TAB. VII
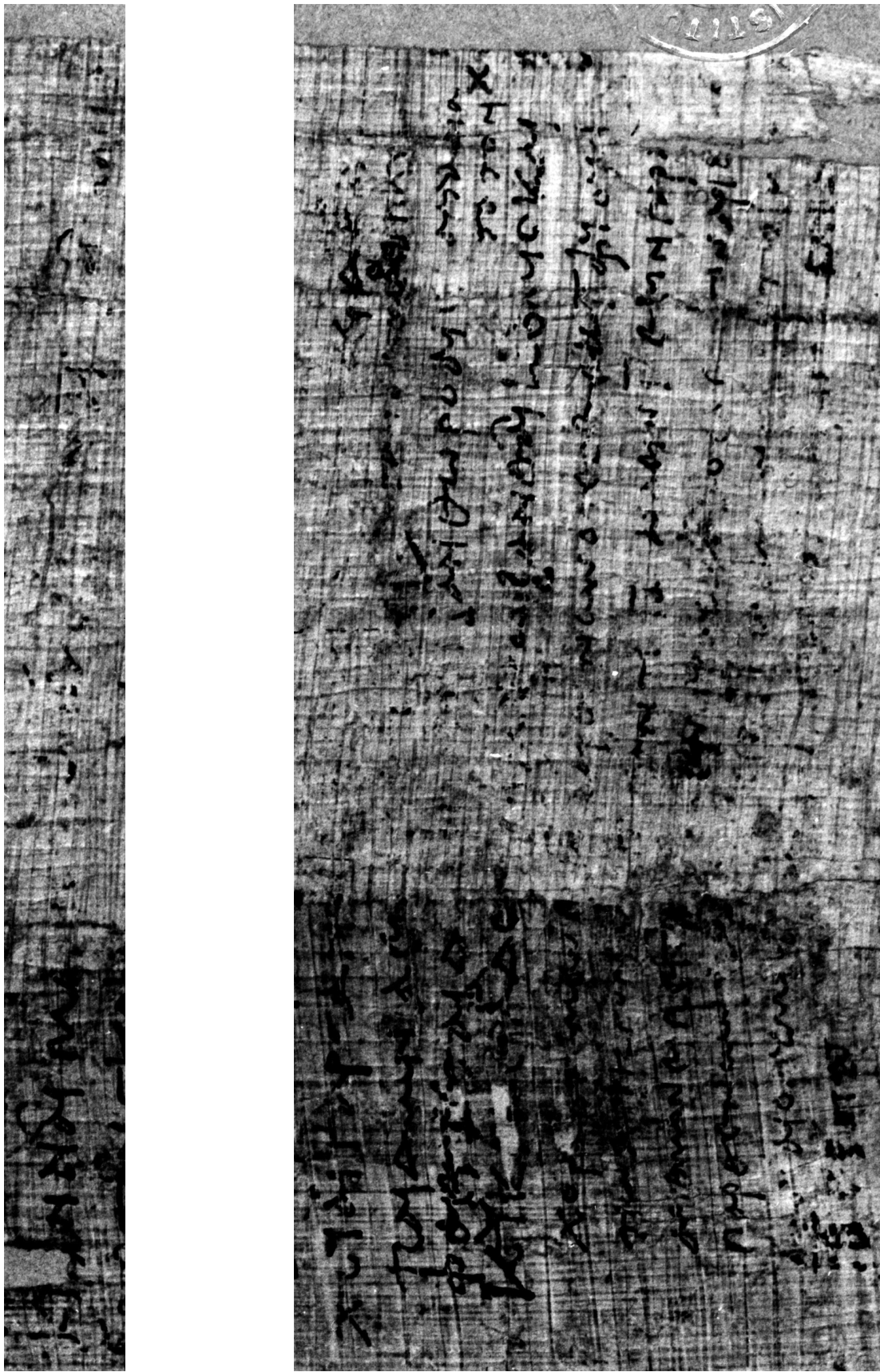

(a) P.Louvre E 3320

(= P.Paris 71),

ad V. $95 \Rightarrow$ Alcman 5

(b) P.Louvre E 3320 (= P.Paris 71), ad V. $98 \Rightarrow$ Alcman 5 
TAB. VIII

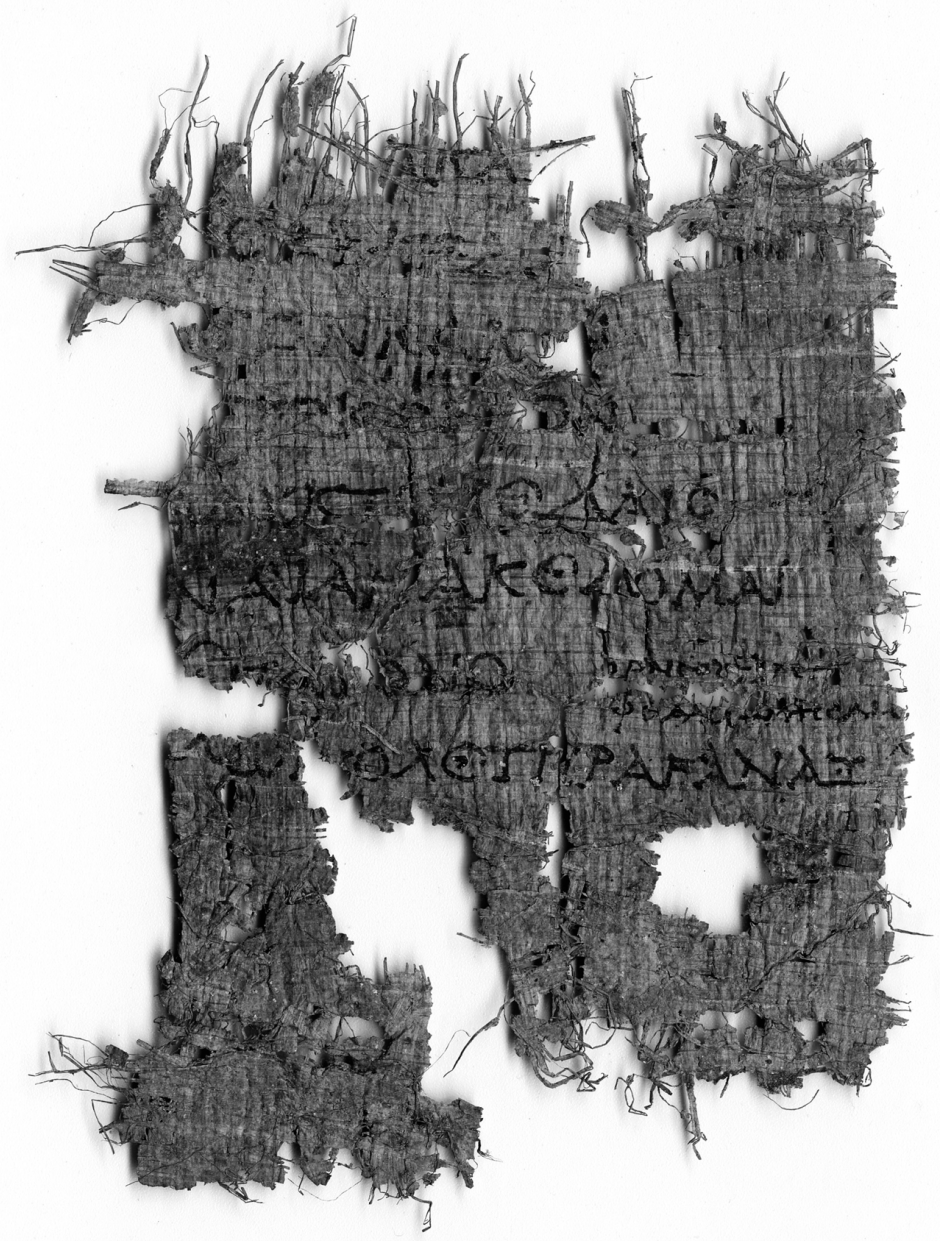

(a) P.Congr.XV $1 \Rightarrow$ Alcman 7

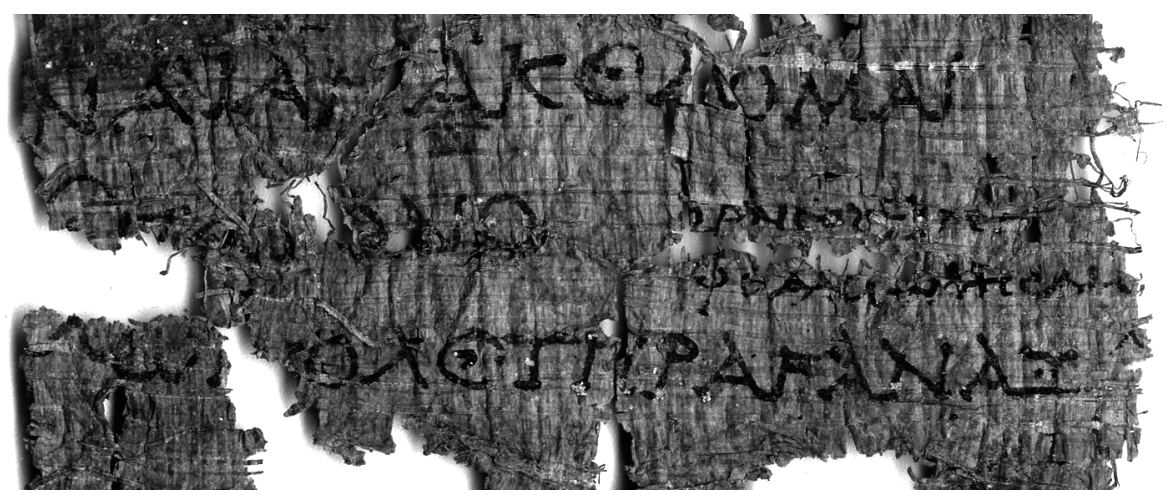

(b) P.Congr.XV $1 \Rightarrow$ Alcman 7 